\title{
NUMERICAL INVESTIGATION OF MECHANICAL BEHAVIOR OF CRACKED CRUCIFORM SPECIMENS IN ALUMINUM ALLOY 6082-T6 SUBJECTED TO DIFFERENT BIAXIAL LOADING CONDITIONS
}

\author{
FoudiL KHELIL \\ Department of Mechanical Engineering, University Mustapha Stambouli, Mascara, Algeria \\ e-mail:khelilfoudil@yahoo.com
}

Benaoumeur Aour

Laboratory of Applied Biomechanics and Biomaterials (LABAB), ENP Oran-MA, Oran, Algeria

ABDERRAHIM TALHA

Ecole des Hautes Etudes d'Ingénieur de Lille, France

Noureddine BenseddiQ

Lille Mechanics Laboratory (LML), University of Lille 1, Villeneuve-d'Ascq, France

\begin{abstract}
Analysis of cracked cruciform specimens under biaxial loading conditions is very important and closer to reality in the study of behavior of marine, naval, aeronautical and railway structures. The aim of this work is to examine the evolution of fracture parameters in a combined mixed mode of an aluminum alloy A6082-T6 cruciform specimen as a function of the biaxial loading with different ratios. To this end, the effects of main parameters, such as load ratio, crack length, crack orientation and non-proportional loading coefficient have been analyzed and discussed in order to highlight fracture toughness of the studied material. The results show that the finite element method is a useful tool for calculation of crack characteristics in the mechanics of biaxial fracture. According to the obtained results, a non-proportional evolution of the fracture parameters, namely, the SIFs $K_{I}$ and $K_{I I}$, T-stress, and the biaxiality parameter was observed. Indeed, the latter depends considerably on the crack length, the angle of crack orientation and the applied biaxial loading. Detailed concluding remarks are presented at the end of this work.
\end{abstract}

Keywords: fracture mechanics, 6082-T6 aluminum alloy, cruciform specimen, finite element analysis, stress intensity factor, T-stress, biaxiality

\section{Introduction}

Fracture is the primary threat to the integrity, safety and performance of nearly all highly stressed mechanical structures in engineering. Failures due to fracture can have major negative consequences. Furthermore, fatigue failure of structural elements subjected to biaxial stress system may develop from surface flaws, and relatively few analyses have been carried out to determine the stress intensity factors along the defects and crack growth rates (Freitas et al., 2014; Hatanaka et al., 1997; Truchon et al., 1981). The effect of notch radius and thickness on the stress concentration and deformation factors in plates with central notch defects subjected to uniaxial and biaxial loading were analyzed using three dimensional finite elements by Abd-Elhady (2014). It was found that the maximum stress and strain concentration factors occurred on the midplane of the plate only in the case of a thin plate. However, the stress and strain concentration factors increased with the decreasing biaxial ratio inside the plate while the opposite trend was found at the plate surface (Pickard, 2015). 
A cruciform biaxial test specimens with seven biaxial tensile tests were conducted on 2219-T87 aluminum alloy by Dawicke and Pollock (1997). In that study an elastic-plastic finite element analysis was used to simulate each test and predict the yield stresses. Other considerations by Shlyannikov et al. (2014), Muhsin et al. (2013) in using the cruciform specimens (CS) included specimen alignment, size and uniformity of the biaxial stress. Tensile tests, both biaxial and uniaxial, require a definition of the yielding. On this basis, crack growth interpretation is performed using the traditional elastic stress intensity factors.

Nevalainen (1997) and Xiao et al. (2015) focused on the relations between crack size on the free surface of a specimen, CTOD and aspect ratio under different fatigue loading conditions. They proved that small-scale yielding correction (SSYC) and statistical treatment for specimen thickness effect are applicable for specimen size correction for actual specimens, while T-stress and SIF seemed to serve better as qualitative indexing parameters.

Many structural applications are subjected to multi-axial states of stress. The estimation of the effect of the first non-singular stress term and the stress intensity factor (SIF) on the fracture behavior of the cruciform specimen under biaxial loading conditions was made by Ayatollahi et al. (2011), Banerjee et al. (2015) and Upadhyay et al. (2017). The results demonstrated that neglecting the first non-singular stress term could lead to significant errors in predicting the apparent fracture toughness of notched components.

The T-stress originally proposed to two-parameter fracture toughness for pure mode I under monotonic/static loading was employed by Shlyannikov et Zakharov (2014) to study the crack growth rate under the cyclic mixed mode fracture. They found that there was a greater variation of the mixed mode crack growth rate depending on the current value of the T-stress along the curvilinear crack trajectories.

Computation of crack characteristics (SIF and T-stress) in three dimensional specimens was presented by Novotný (2012) and Navarro-Zafra et al. (2016). The SIF was determined by processing displacements around the crack tip. Different methods have been used for calculating the T-stress. Cruciform specimens with an inclined crack subject to biaxial loads to study the fatigue crack growth rate under the mixed mode were reported by many authors (Eftis and Subramonian, 1978; Lamkanfi et al., 2010). They suggested a method for infiltrating the mixed mode displacement of cracks in the deformed state.

In this paper, numerical results for different crack lengths and different angles of the crack orientation in cruciform specimens under different biaxial loading conditions are presented. Indeed, the evolution of the fracture parameters such as, $K_{I}, K_{I I}$, T-stress and the biaxiality parameter as a function of crack length, loading conditions and the crack orientation angle has been highlighted and discussed.

\section{Background formulation}

The Williams approach proposes a general alternative resolution of equation (2.1) without using complex functions. We assume for this that the Airy function $A$ is written in the form

$$
A(r, \alpha)=r^{\gamma+1} F(\alpha)
$$

where $\gamma$ is a scalar, $r$ is the radial distance from the crack tip, $\alpha$ is an angular coordinate and $F(\alpha)$ a continuous and differentiable function of $\alpha$ to be determined.

As shown by Pickard (1986), the governing equation of linear elasticity problems is a two-dimensional bi-harmonic equation in terms of function $A(r, \alpha)$. Accordingly, the differential equation is expressed in cylindrical coordinates as

$$
\left(\frac{\delta^{2}}{\delta r^{2}}+\frac{1}{r} \frac{\delta}{\delta r}+\frac{1}{r^{2}} \frac{\delta^{2}}{\delta \alpha^{2}}\right)\left(\frac{\delta^{2} A}{\delta r^{2}}+\frac{1}{r} \frac{\delta A}{\delta r}+\frac{1}{r^{2}} \frac{\delta^{2} A}{\delta \alpha^{2}}\right)=0
$$


By substituting the previous expression of $A(r, \alpha)$ into equation (2.2), we obtain the following 4-th order differential equation for $F(\alpha)$

$$
\frac{d^{4} F(\alpha)}{d \alpha^{4}}+\left[(\gamma-1)^{2}+(\gamma+1)^{2}\right] \frac{d^{2} F(\alpha)}{d \alpha^{2}}+(\gamma-1)^{2}(\gamma+1)^{2} F(\alpha)=0
$$

The general solution for $F(\alpha)$ which satisfies Eq. (2.3) is

$$
F(\alpha)=A \cos (\gamma+1) \alpha+B \cos (\gamma+1) \alpha+C \sin (\gamma-1) \alpha+D \sin (\gamma-1) \alpha
$$

where $A, B, C$ and $D$ are constants which will be set by boundary conditions.

The stresses are related to the Airy stress function by the following expressions (Pickard, 1986)

$$
\sigma_{r r}=\frac{1}{r} \frac{\partial A}{\partial r}+\frac{1}{r^{2}} \frac{\partial A}{\partial \alpha^{2}} \quad \sigma_{\alpha \alpha}=\frac{\partial^{2} A}{\partial r^{2}} \quad \sigma_{r \alpha}=\frac{1}{r^{2}} \frac{\partial A}{\partial \alpha}-\frac{1}{r} \frac{\partial^{2} A}{\partial \alpha \partial r}
$$

Substitution of equation (2.1) into (2.5) yields

$$
\begin{aligned}
& \sigma_{r r}=r^{(\gamma-1)}\left[\frac{d^{2} F(\alpha)}{d \alpha^{2}}+(\gamma+1) F(\alpha)\right] \\
& \sigma_{\alpha \alpha}=r^{(\gamma-1)} \gamma(\gamma+1) F(\alpha) \quad \sigma_{r \alpha}=-r^{(\gamma-1)} \gamma \frac{d F(\alpha)}{d \alpha}
\end{aligned}
$$

where $\sigma_{r r}, \sigma_{\alpha \alpha}$ and $\sigma_{r \alpha}$ are the stresses components in cylindrical coordinates.

Finding a solution for $F(\alpha)$ requires that

$$
F(0)=F(2 \pi)=0 \quad \frac{d F(0)}{d \alpha}=\frac{d F(2 \pi)}{d \alpha}=0
$$

These boundary conditions are satisfied if

$$
\begin{aligned}
& A=-C \quad B=-\frac{\gamma-1}{\gamma+1} D \\
& \sin (2 \pi \gamma)=0 \quad \gamma=\frac{n}{2} \quad n=1,2,3, \ldots
\end{aligned}
$$

There is a corresponding function $A_{n}(r, \alpha)$ for each value of $\gamma, \gamma_{n}$, which satisfies the biharmonic equation and the applied boundary conditions. The most general solution is obtained by taking a linear combination of these solutions

$$
A(r, \alpha)=\sum_{n=1}^{\infty} r^{\frac{n}{2}+1} F_{n}(\alpha)
$$

where

$$
\begin{aligned}
& F_{n}(\alpha)=C_{n}\left[\cos \left(\frac{n}{2}+1\right)(\alpha+\pi)-\cos \left(\frac{n}{2}+1\right)(\alpha+\pi)\right] \\
& \quad+D_{n}\left[\sin \left(\frac{n}{2}-1\right)(\alpha+\pi)+\frac{n-2}{n+2} \sin \left(\frac{n}{2}+1\right)(\alpha+\pi)\right]
\end{aligned}
$$

From equation (2.6), and by considering only the crack tip region $(0<r \ll 1)$, the term in $\sqrt{r}$ above becomes relatively insignificant. Thus the crack tip stress field, up to the first three terms, expressed in Cartesian coordinates is of the form

$$
\begin{aligned}
\sigma_{11} & =\frac{1}{\sqrt{r}}\left[-D_{1} \cos \frac{\alpha}{2}\left(1-\sin \frac{\alpha}{2} \sin \frac{3 \alpha}{2}\right)-C_{1} \sin \frac{\alpha}{2}\left(2+\cos \frac{\alpha}{2} \cos \frac{3 \alpha}{2}\right)\right]+C_{2} \\
\sigma_{22} & =\frac{1}{\sqrt{r}}\left[C_{1} \sin \frac{\alpha}{2} \cos \frac{\alpha}{2} \cos \frac{3 \alpha}{2}-D_{1} \cos \frac{\alpha}{2}\left(1+\sin \frac{\alpha}{2} \sin \frac{3 \alpha}{2}\right)\right] \\
\sigma_{12} & =\frac{1}{\sqrt{r}}\left[C_{1} \cos \frac{\alpha}{2}\left(1-\sin \frac{\alpha}{2} \sin \frac{3 \alpha}{2}\right)-D_{1} \sin \frac{\alpha}{2} \cos \frac{\alpha}{2} \cos \frac{3 \alpha}{2}\right]
\end{aligned}
$$


Now, by considering the Westergaard method, the stress field can be expressed in complex space as

$$
\begin{aligned}
& \sigma_{11}=\operatorname{Re}\left[2 \xi^{\prime}(z)\right]-x_{2} \operatorname{Im}\left[2 \xi^{\prime \prime}(z)\right]+\beta \\
& \sigma_{22}=\operatorname{Re}\left[2 \xi^{\prime}(z)\right]+x_{2} \operatorname{Im}\left[2 \xi^{\prime \prime}(z)\right]-\beta \\
& \sigma_{12}=-x_{2} \operatorname{Im}\left[2 \xi^{\prime \prime}(z)\right]+\beta
\end{aligned}
$$

where $z=x_{1}+\mathrm{i} x_{2}=r \mathrm{e}^{\mathrm{i} \alpha}$ is a complex number, $\xi(z)$ is an analytical function termed "complex potential" and $\beta$ is a real function of $x_{1}$ and $x_{2}$.

For the crack tip region, it can be demonstrated that (Pickard, 1986)

$$
2 \xi^{\prime}(z) \approx \frac{\sqrt{a} \sigma}{\sqrt{2(z-a)}}-\frac{(1-k) \sigma}{2}=\frac{\sqrt{a} \sigma}{\sqrt{2 r}}\left(\cos \frac{\alpha}{2}-\mathrm{i} \sin \frac{\alpha}{2}\right)-\frac{(1-k) \sigma}{2}
$$

and

$$
2 \xi^{\prime \prime}(z) \approx \frac{\sqrt{a} \sigma}{2 \sqrt{[2(z-a)]^{3}}}=\frac{\sqrt{a} \sigma}{\sqrt{(2 r)^{3}}}\left(\cos \frac{3 \alpha}{2}-\mathrm{i} \sin \frac{3 \alpha}{2}\right)
$$

Substituting into equations (2.12), with the value of $\beta=-(1-k) \sigma / 2$, gives

$$
\begin{aligned}
\sigma_{11} & =\frac{K_{I}}{\sqrt{2 \pi r}} \cos \frac{\alpha}{2}\left(1-\sin \frac{\alpha}{2} \sin \frac{3 \alpha}{2}\right)-(1-k) \sigma \\
\sigma_{22} & =\frac{K_{I}}{\sqrt{2 \pi r}} \cos \frac{\alpha}{2}\left(1+\sin \frac{\alpha}{2} \sin \frac{3 \alpha}{2}\right) \\
\sigma_{12} & =\frac{K_{I}}{\sqrt{2 \pi r}} \cos \frac{\alpha}{2} \sin \frac{\alpha}{2} \cos \frac{3 \alpha}{2}
\end{aligned}
$$

where $K_{I}=\sigma \sqrt{\pi a}$ is defined as the mode I stress intensity factor and $k=\sigma_{x x} / \sigma_{y y}$ is the nominal stress ratio.

It should be noted that this analysis was first performed by Westergaard for equibiaxial loading, and was subsequently expanded to cover the general biaxial loading by Sih (1966).

By making comparison with Eq. (2.11), we get

$$
D_{1}=-\frac{K_{I}}{\sqrt{2 \pi}}=-\sigma \sqrt{\frac{a}{2}} \quad C_{2}=-(1-k) \sigma
$$

A similar analysis, for shear loading of the remote boundaries, leads to

$$
C_{1}=-\frac{K_{I I}}{\sqrt{2 \pi}}=\tau \sqrt{\frac{a}{2}}
$$

where $K_{I I}=\tau \sqrt{\pi a}$ is defined as the mode II stress intensity factor, for remote shear stress $\tau$.

Consequently, the general expressions for the stresses in the vicinity of the crack tip (2.11) can be now written as

$$
\begin{aligned}
\sigma_{11} & =\frac{K_{I}}{\sqrt{2 \pi r}} \cos \frac{\alpha}{2}\left(1-\sin \frac{\alpha}{2} \sin \frac{3 \alpha}{2}\right)-\frac{K_{I I}}{\sqrt{2 \pi r}} \sin \frac{\alpha}{2}\left(2+\cos \frac{\alpha}{2} \cos \frac{3 \alpha}{2}\right)+C_{2} \\
\sigma_{22} & =\frac{K_{I}}{\sqrt{2 \pi r}} \cos \frac{\alpha}{2}\left(1+\sin \frac{\alpha}{2} \sin \frac{3 \alpha}{2}\right)+\frac{K_{I I}}{\sqrt{2 \pi r}} \sin \frac{\alpha}{2} \cos \frac{\alpha}{2} \cos \frac{3 \alpha}{2} \\
\sigma_{12} & =\frac{K_{I}}{\sqrt{2 \pi r}} \sin \frac{\alpha}{2} \cos \frac{\alpha}{2} \cos \frac{3 \alpha}{2}+\frac{K_{I I}}{\sqrt{2 \pi r}} \cos \frac{\alpha}{2}\left(1-\sin \frac{\alpha}{2} \sin \frac{3 \alpha}{2}\right)
\end{aligned}
$$

where $C_{2}=-(1-k) \sigma, K_{I}=\sigma \sqrt{\pi a}$ and $K_{I I}=\tau \sqrt{\pi a}$ for the general remote loading of a crack length $2 a$ in an infinite sheet. 
Now, consider that the crack makes an angle $\theta$ with the $y$ direction in an infinite plate subjected to stresses $\sigma$ and $k \sigma$ along the $y$ and $x$ direction, respectively, at infinity (Fig. 1a). By stress transformation, we obtain the following stresses $\sigma_{x}^{\prime}, \sigma_{y}^{\prime}, \tau_{x y}^{\prime}$ in the system $x^{\prime} y^{\prime}$ (Fig. 1b)

$$
\begin{array}{rlr}
\sigma_{x}^{\prime} & =\frac{k+1}{2} \sigma-\frac{k-1}{2} \sigma \cos 2 \theta \quad \sigma_{y}^{\prime}=\frac{k+1}{2} \sigma+\frac{k-1}{2} \sigma \cos 2 \theta \\
\tau_{x y}^{\prime} & =-\frac{k-1}{2} \sigma \sin 2 \theta &
\end{array}
$$

Thus, the stress field at the crack tip is obtained by superposing an opening-mode loading caused by the stress $\sigma_{y}^{\prime}$ and a sliding-mode loading caused by the stress $\tau_{x y}^{\prime}$.
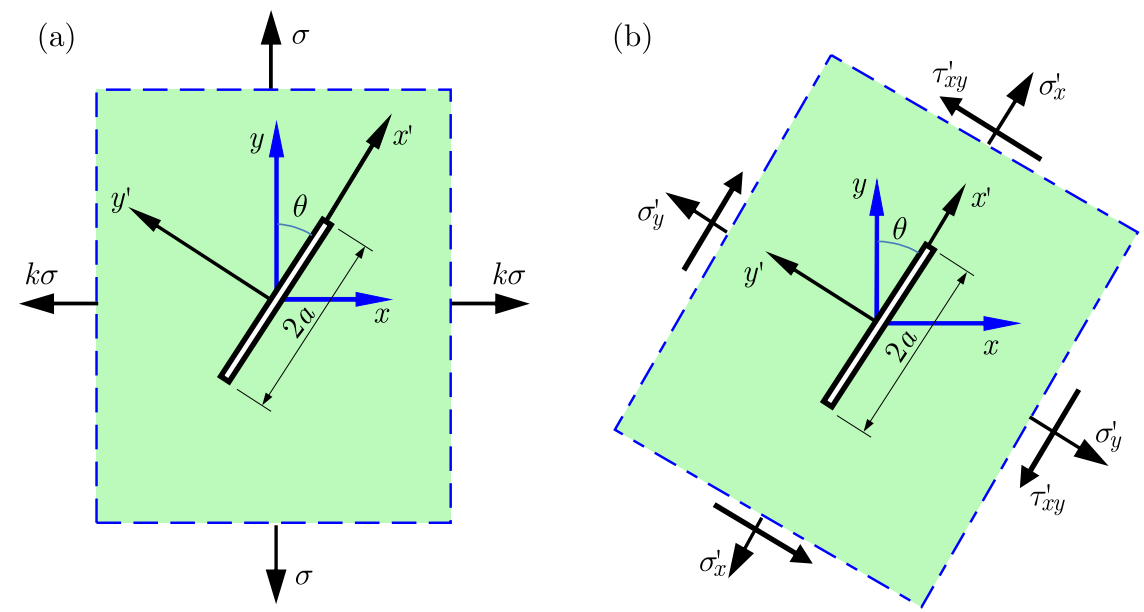

Fig. 1. An inclined crack (a) in a biaxial stress field and (b) stress transformation along and perpendicular to the crack plane

The stress $\left(\sigma_{y}^{\prime}-\sigma_{x}^{\prime}\right)$ does not create singular stresses but should be subtracted from the $\sigma_{x}^{\prime}$ stress along the $x^{\prime}$-axis (Gdoutos, 2005). From Eqs. (2.18) and (2.19), we obtain the stresses

$$
\begin{aligned}
& \sigma_{x^{\prime}}=\frac{K_{I}}{\sqrt{2 \pi r}} \cos \frac{\alpha}{2}\left(1-\sin \frac{\alpha}{2} \sin \frac{3 \alpha}{2}\right)-\frac{K_{I I}}{\sqrt{2 \pi r}} \sin \frac{\alpha}{2}\left(2+\cos \frac{\alpha}{2} \cos \frac{3 \alpha}{2}\right)+T \\
& \sigma_{y^{\prime}}=\frac{K_{I}}{\sqrt{2 \pi r}} \cos \frac{\alpha}{2}\left(1+\sin \frac{\alpha}{2} \sin \frac{3 \alpha}{2}\right)+\frac{K_{I I}}{\sqrt{2 \pi r}}\left(\sin \frac{\alpha}{2} \cos \frac{\alpha}{2} \cos \frac{3 \alpha}{2}\right) \\
& \tau_{x^{\prime} y^{\prime}}=\frac{K_{I}}{\sqrt{2 \pi r}} \sin \frac{\alpha}{2} \cos \frac{\alpha}{2} \cos \frac{3 \alpha}{2}+\frac{K_{I I}}{\sqrt{2 \pi r}} \cos \frac{\alpha}{2}\left(1-\sin \frac{\alpha}{2} \sin \frac{3 \alpha}{2}\right)
\end{aligned}
$$

where $K_{I}$ and $K_{I I}$ take the following form

$$
K_{I}=\frac{\sigma \sqrt{\pi a}}{2}[(1+k)-(1-k) \cos 2 \theta] \quad K_{I I}=\frac{\sigma \sqrt{\pi a}}{2}[(1-k) \sin 2 \theta]
$$

and $T$ is the T-stress which is given by

$$
T=\sigma(1-k) \cos 2 \theta
$$

In the case of a specimen with finite dimensions, expression (2.21) becomes

$$
K_{I}=\frac{\sigma \sqrt{\pi a}}{2}[(1+k)-(1-k) \cos 2 \theta] Y_{I} \quad K_{I I}=\frac{\sigma \sqrt{\pi a}}{2}[(1-k) \sin 2 \theta] Y_{I I}
$$

where $Y_{I}$ and $Y_{I I}$ are functions which take into account the influence of specimen geometry, the load biaxiality ratio and the crack angle. 
The J-integral for the mixed mode loading can be expressed as a function of the elastic stress intensity factors $K_{I}$ and $K_{I I}$ as follows (Shlyannikov, 2013)

$$
\begin{aligned}
& J_{x}=\frac{(1+\nu)(1+\kappa)}{4 E}\left(K_{I}^{2}+K_{I I}^{2}\right) \quad J_{y}=\frac{(1+\nu)(1+\kappa)}{2 E} K_{I} K_{I I} \\
& J=\sqrt{J_{x}^{2}+J_{y}^{2}}
\end{aligned}
$$

where $\kappa=3-4 \nu$ for plane strain, $\kappa=(3-\nu) /(1+\nu)$ for plane stress, $\nu$ and $E$ are Poisson's coefficient and Young's modulus, respectively.

\section{Geometrical model and material properties}

The cruciform specimen made of 6082-T6 aluminum alloy (Fig. 2) was designed according to criteria announced by Dawicke and Pollock (1997). Symmetrical boundary conditions were considered. The plate was loaded by a load $\sigma$ in the $y$-direction, and loaded in the $x$-direction by $\sigma_{x x}=k \sigma_{y y}$ at plate edges as shown in Fig. 2. $\sigma$ varied from 100 to $900 \mathrm{MPa}$, and $k$ is the nominal stress ratio.

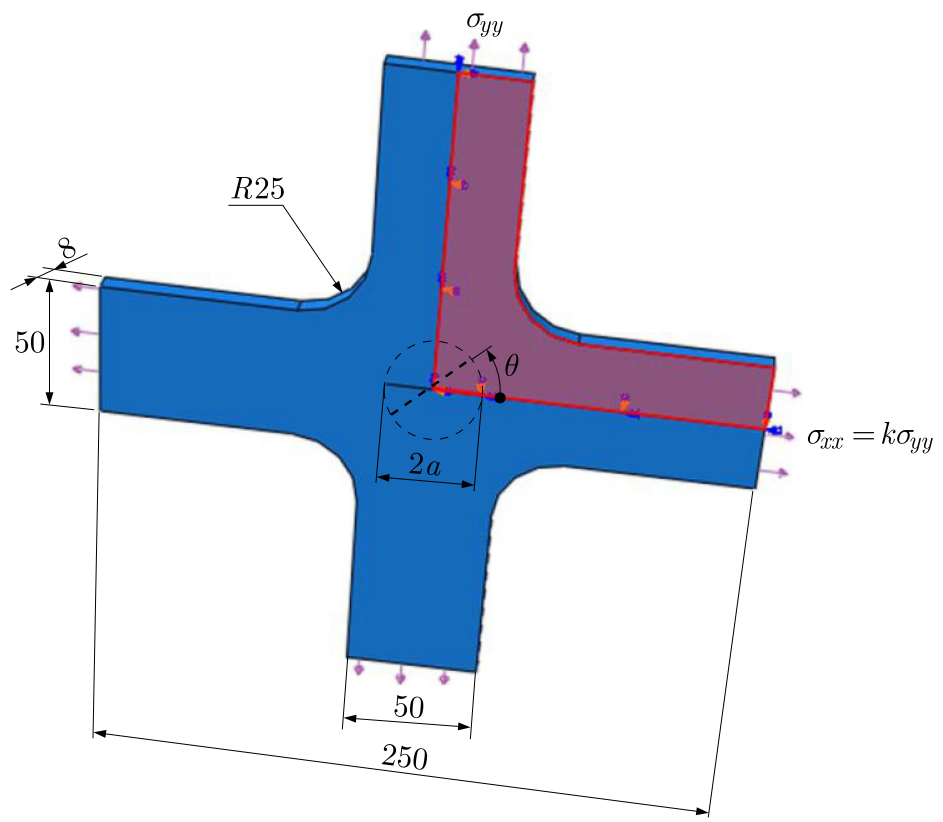

Fig. 2. Illustration of the specimen geometry with the boundary conditions and loading

The mechanical properties of the plate were as follows: modulus of elasticity $E=77000 \mathrm{MPa}$, Poisson's ratio $\nu=0.3$. As illustrated in Fig. 2, the plate was $240 \mathrm{~mm}$ in height, $240 \mathrm{~mm}$ in width, and had thickness of $8 \mathrm{~mm}$.

In the first part, the crack length ranged from $5 \mathrm{~mm}$ to $25 \mathrm{~mm}$ with a step of $5 \mathrm{~mm}$, and a horizontal crack $(\theta=0)$ was chosen.

In the second phase, the crack length was $10 \mathrm{~mm}$ and the angle of crack orientation $\theta$ varied from $0^{\circ}$ to $90^{\circ}$ with a step of $15^{\circ}$. The plate was analyzed for the bi-equiaxial loading by applying stress in the $x$ - and $y$-axis directions with $\sigma_{x x}=k \sigma_{y y}$ uniformly applied to its ends as shown in Fig. 2. In order to highlight the effect of loading conditions on the evolution of the fracture parameters, different values of $k$ were tested. 


\section{Finite element modeling}

Three dimensional finite element models of cruciform specimens were developed using ABAQUS software (2013). It is difficult to obtain an acceptable mesh using global seeding. Then, the specimen was discretized in several subdomains according to the angular direction of the crack. Each subdomain was meshed individually using a refined mesh with 3D linear hexadecimal elements (C3D8). The results obtained with the elements C3D8 were more numerically accurate than those obtained with C3D8R (reduced integration elements).

Figure 3 shows a typical FE mesh modeling of the cruciform specimen under consideration. A highly refined mesh in the crack-tip region as shown in Figs. 3b and 3c was used to provide a good resolution of stress fields over the normalized distance at the crack tip. Regular integration and a collapsed element side with the duplicate node technique were employed to eliminate shear locking in the elements. We note that this method is coherent and cost-effective for very specific calculation of the fracture parameters (SIF, T-stress and the biaxiality parameter).

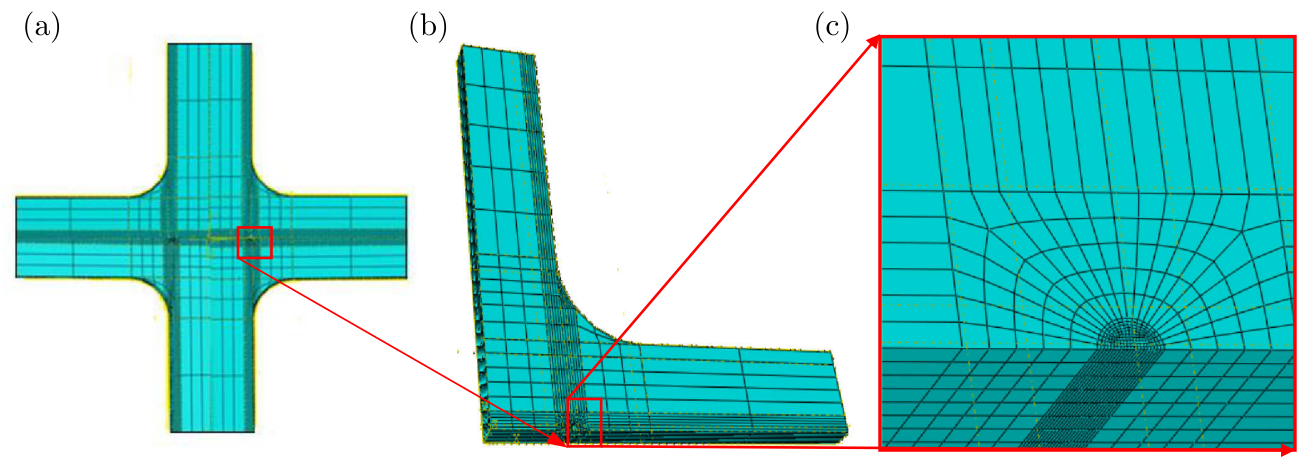

Fig. 3. Illustration of: (a) finite element model employed, (b) typical mesh used for a quarter of the cruciform specimen, (c) detail of the refined mesh near the crack tip

The model consists of 25690 linear hexahedral elements and 29513 nodes arranged in a focused or centered fan configuration at the crack tip. It should be noted that for crack orientation analysis, in the second part of this study, the mesh was refined and oriented according to the crack angle.

\section{Results and discussion}

The aim of this work is to study the evolution of different fracture parameters in three-dimensional finite elements in the case of a cracked cruciform specimen made of 6082-T6 aluminum alloy. Consequently, details of the models for evaluation of the fracture parameters under the biaxial loading and various cracks orientations in the mixed mode will be discussed in this work.

\subsection{Evolution of SIF}

Using a simple definition which requires no additional assumptions, the elastic stress intensity factors can also characterize stress fields at the crack tip and control of 3D fracture. Figures 4 and 5 show respectively the evolution of $K_{I}$ and $K_{I I}$ factors as a function of the applied load for different crack lengths at the center of the cruciform specimen. The decisive argument for the choice of the fracture parameters is the sensitivity of $K_{I}$ and $K_{I I}$ through the stresses effects. Plane strain conditions have been assumed in the numerical simulations. 
In the case of $\sigma_{y y}=100 \mathrm{MPa}$ and varied $\sigma_{x x}$ (Fig. 4a), it can be seen that the SIF decreases linearly with the increase of the horizontal applied load $\sigma_{x x}$. This can be attributed to the crack lips closing due to the loading parallel to them.

(a)

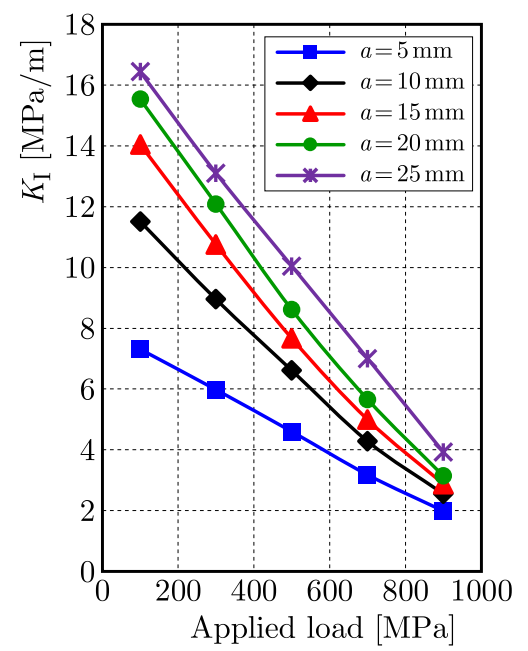

(b)

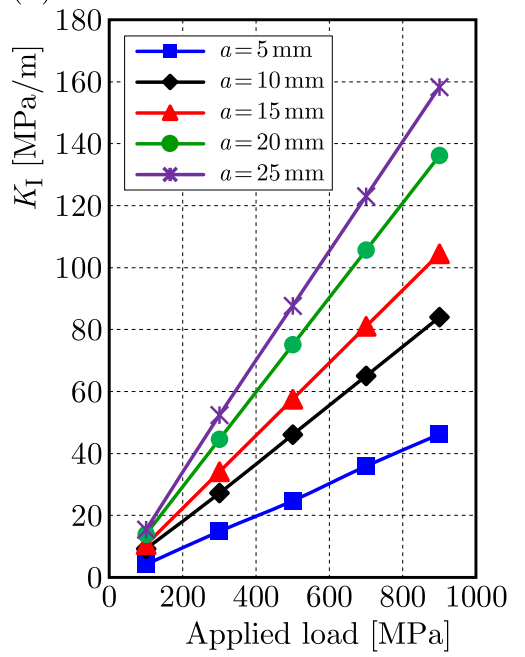

(c)

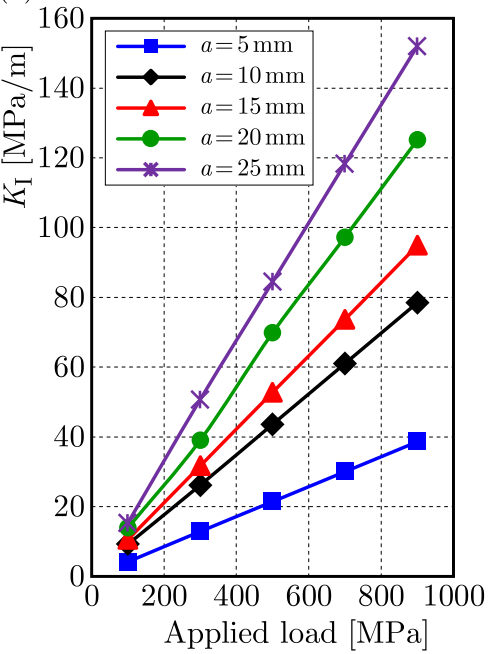

Fig. 4. Evolution of $K_{I}$ versus the applied loading for different crack lengths in the mixed mode in the case of: (a) $\sigma_{y y}=100 \mathrm{MPa}$ and varied $\sigma_{x x}$, (b) $\sigma_{x x}=100 \mathrm{MPa}$ and varied $\sigma_{y y}$ and (c) with varied bi-equiaxial tensile load

In the case of $\sigma_{x x}=100 \mathrm{MPa}$ and varied $\sigma_{y y}$ (Fig. 4b), it can be seen that the SIF increases linearly with the increase of the vertical applied load $\sigma_{y y}$. This seems to be in good agreement with the literature since this type of loading contributes to the opening of the crack.

In the case of a varied bi-equiaxial tensile load (Fig. 4c), it can be noted that the SIF increases linearly with the increase of the applied load in a somewhat weak way compared with the previous case. This explains the theoretical relationship between $K_{I}$ and the load biaxiality ratio $k$ (which is given by the ratio: $\sigma_{y y} / \sigma_{x x}$ ) (Ayatollahi et al., 1998; Papadopoulos 1988). Furthermore, it should be noted generally that the SIF increases with the increase of the crack length for the three cases.

(a)

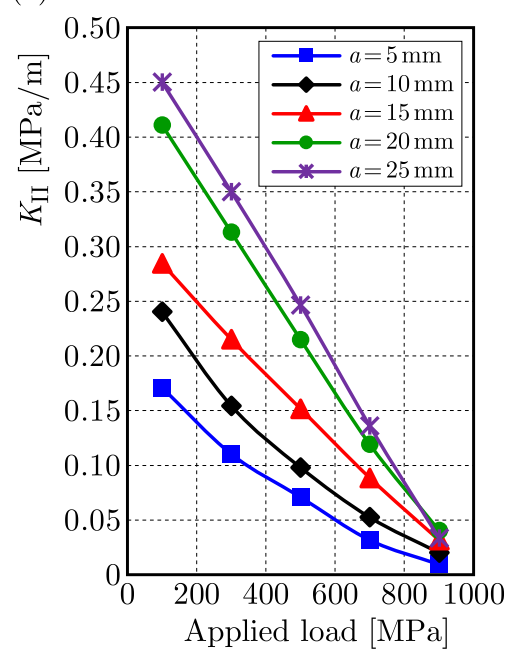

(b)

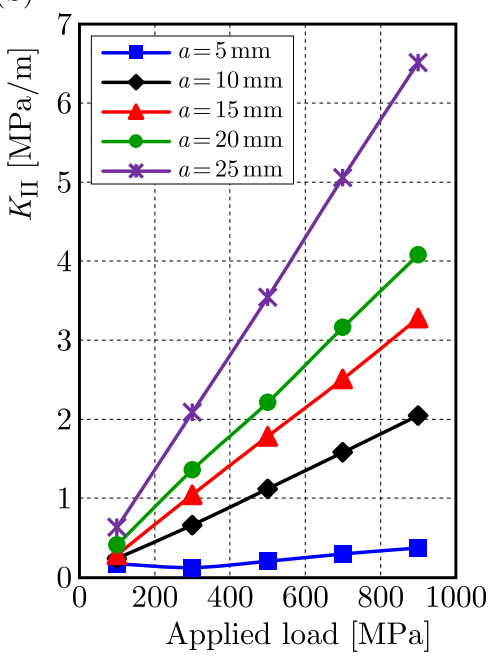

(c)

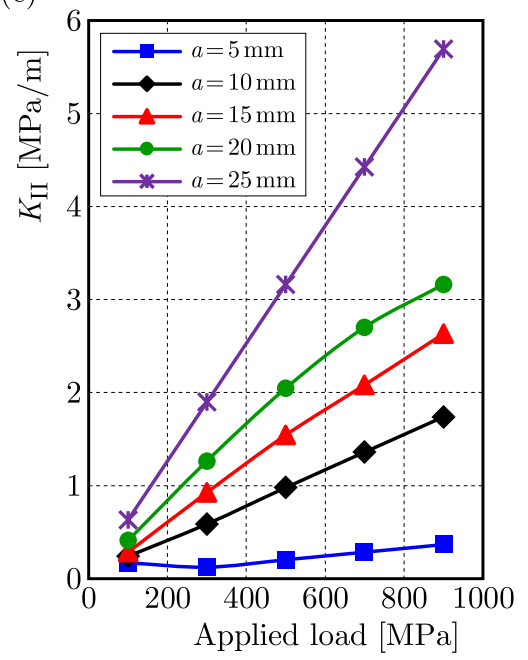

Fig. 5. Evolution of $K_{I I}$ versus the applied loading for different crack lengths in the mixed mode in the case of: (a) $\sigma_{y y}=100 \mathrm{MPa}$ and varied $\sigma_{x x}$, (b) $\sigma_{x x}=100 \mathrm{MPa}$ and varied $\sigma_{y y}$ and (c) with varied bi-equiaxial tensile load 
The same trends of variation have been found in the case of $K_{I I}$ as shown in Fig. 5. The only difference lies in the magnitude of the values obtained which are lower compared to those of $K_{I}$. It should also be noted that $K_{I I}$ is not zero even though the crack is horizontal. This means that the crack failure mode under consideration is not a pure mode $\mathrm{I}$.

\subsection{Evolution of T-stress}

The T-stress has been recognized to be a measure of constraint for small-scale yielding conditions. It is further assumed that for moderate small-scale yielding conditions or plastic deformations, the biaxial fracture process can be controlled by the single parameter T-stress based on the elastic numerical solutions and, therefore, is reflected in the influence of cracked body geometry and loading conditions. In the present study, the T-stress is employed to analyze the mechanical behavior of the CS under the mixed mode of fracture.

Few methods have been proposed for calculating $T$, among which, we can quote the stress method by Rice (1974), the interaction integral method by Nakamura and Parks (1992), the displacement method by Ayatollahi et al. (1998), the strain gage method Khelil et al. (2017), and others (Shlyannikov et al., 2014). In this study, we explore the direct use of FEM analysis for calculating the T-stress on the basis of crack flank nodal displacements (Ayatollahi et al., 1998).
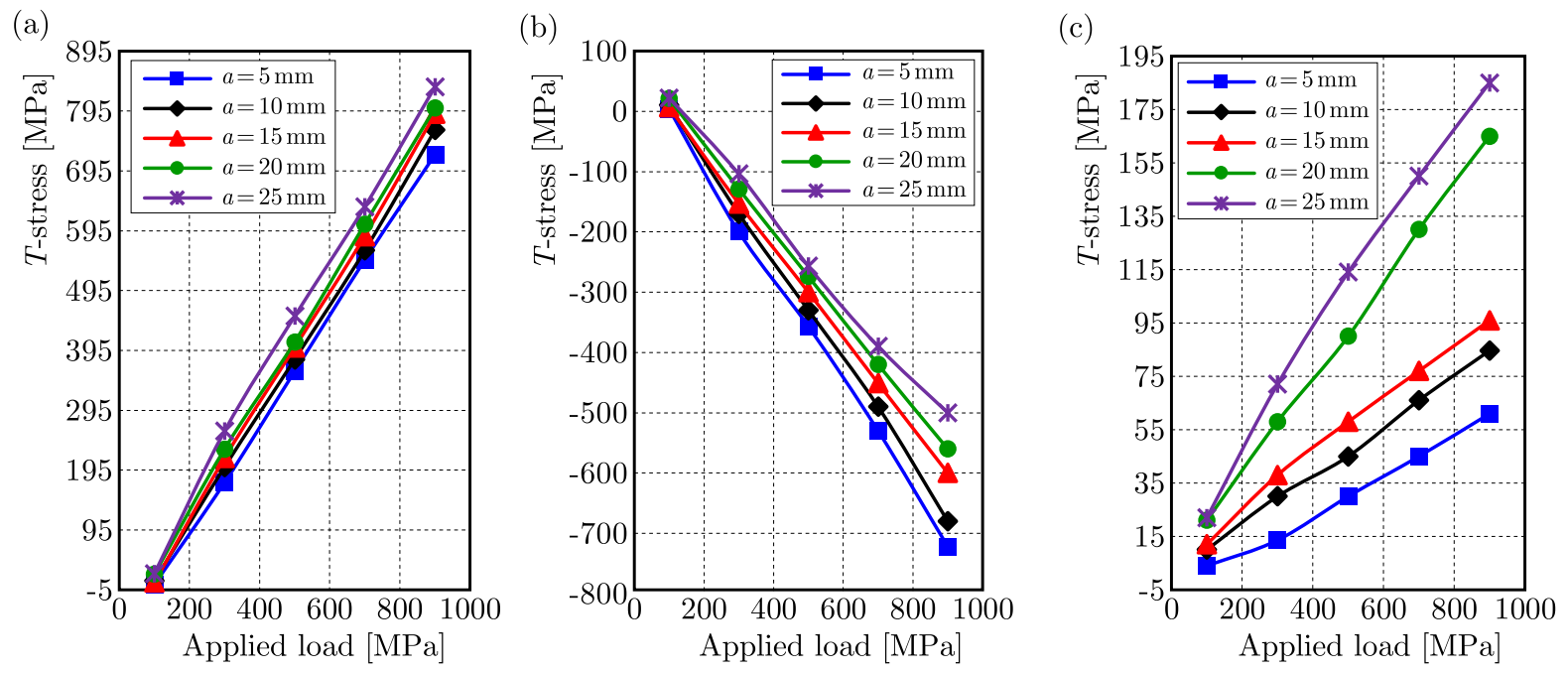

Fig. 6. Evolution of T-stress versus the applied loading for different crack lengths in the mixed mode in the case of: (a) $\sigma_{y y}=100 \mathrm{MPa}$ and varied $\sigma_{x x}$, (b) $\sigma_{x x}=100 \mathrm{MPa}$ and varied $\sigma_{y y}$ and (c) with varied bi-equiaxial tensile load

To this end, the commercial finite element ABAQUS software (2013), has been used to calculate the stress distributions ahead of the crack tip. It should be noted that the calculation of the T-stress was carried out from the values of the same interval as in the calculation of the stress intensity factors. In Fig. 6, the evolution history of the T-stress parameter with the loading is displayed for the horizontal cracked cruciform specimen when the crack length is ranging from $5 \mathrm{~mm}$ to $25 \mathrm{~mm}$. It can be seen that:

- In the case of $\sigma_{y y}=100 \mathrm{MPa}$ and varied $\sigma_{x x}$ (Fig. 6a), the T-stress increases linearly with the increase of the horizontal applied load $\sigma_{x x}$ (with positive values). Indeed, in this case, the non-singular term of the Williams stresses field is a tensile stress and causes closing of the crack lips, which justifies the positive values.

- In the case of $\sigma_{x x}=100 \mathrm{MPa}$ and varied $\sigma_{y y}$ (Fig. 6b), the absolute value of T-stress increases linearly with the increase of the vertical applied load $\sigma_{y y}$. The negative values of 
the latter can be justified by the way that the non-singular term of the Williams stresses field is a compressive stress which can cause the opening of the crack lips.

- In the case of varied bi-equiaxial tensile load (Fig. 6c), the T-stress increases linearly with the increase of the applied load as in the first case, the only difference is the magnitude which is relatively low.

Furthermore, it should be generally noted that the effect of crack length is more pronounced in the third case compared to the first two cases. Based on the results of static biaxial loading fracture, the T-stress is a linear-elastic parameter best suited for constraint indexing.

\subsection{Evolution of the biaxiality parameter}

To characterize the stress effect described by the T-stress with respect to the stress intensity factors, Leevers and Radon (1982) introduced an additional fracture parameter called the local biaxiality ratio $\beta$. In the case of mode I, this parameter is given by the following expression

$$
\beta=\frac{T \sqrt{\pi a}}{K_{I}}
$$

where $K_{I}$ is the stress intensity factor and $a$ is the crack length. Equation (5.1) can be extended to the mixed mode if $K_{I}$ is replaced by an equivalent stress intensity factor $K_{\text {eff }}$ (Leevers and Radon, 1982). Then, the local biaxiality ratio $\beta$ for general mixed mode conditions is

$$
\beta=\frac{T \sqrt{\pi a}}{K_{\text {eff }}}
$$

with

$$
K_{\text {eff }}=\sqrt{K_{I}^{2}+K_{I I}^{2}}
$$

For the same specimen configuration and different crack lengths, the biaxiality parameter $\beta$ has been analyzed as a function of the applied biaxial loading using 3D elastic finite element computations. The obtained results are shown in Fig. 7. It was found that in the first case where $\sigma_{y y}=100 \mathrm{MPa}$ and varied $\sigma_{x x}$ (Fig. 7a), a parabolic variation was observed. The more

(a)

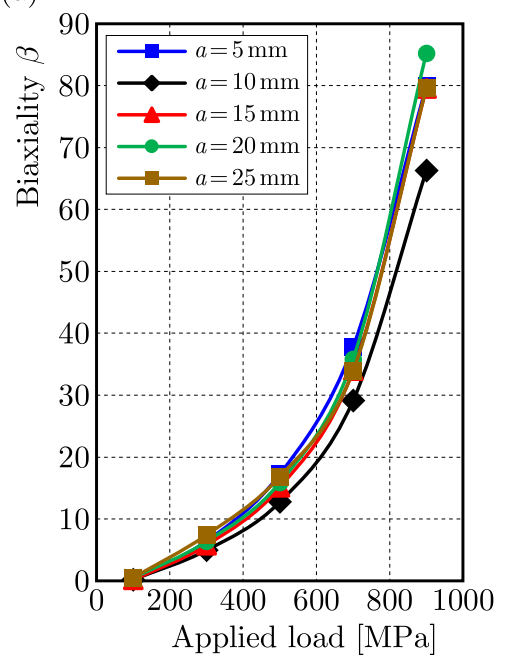

(b)

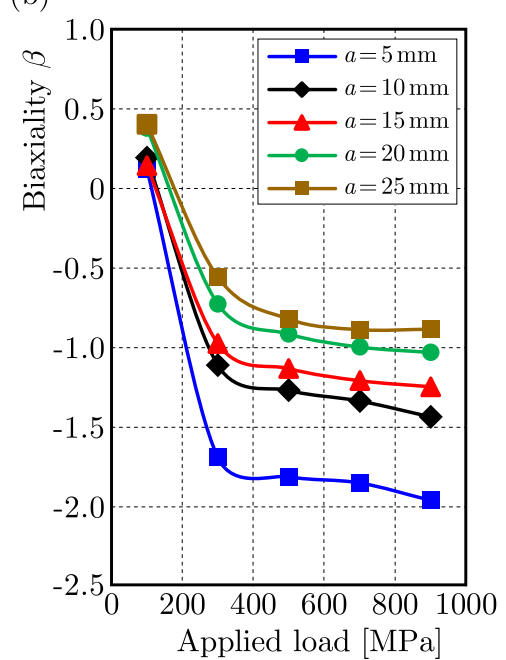

(c)

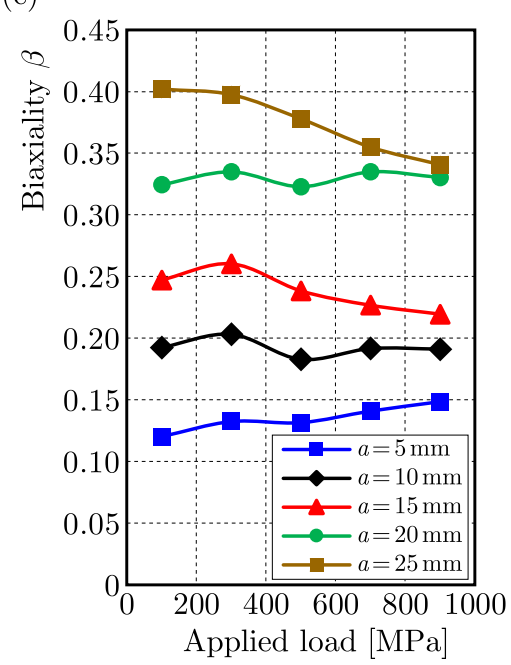

Fig. 7. Evolution of the biaxiality paramters $\beta$ versus the applied loading for different crack lengths in the mixed mode in the case of: (a) $\sigma_{y y}=100 \mathrm{MPa}$ and varied $\sigma_{x x}$, (b) $\sigma_{x x}=100 \mathrm{MPa}$ and varied $\sigma_{y y}$ and (c) with varied bi-equiaxial tensile load 
the applied load increases, the more the parameter $\beta$ increases. In contrast, for the effect of crack length, when $a$ is ranging from $5 \mathrm{~mm}$ to $15 \mathrm{~mm}$, the biaxiality parameter increases with the increase of the latter, exceeding $a=15 \mathrm{~mm}, \beta$ decreases again.

This can be attributed to triple effects of the applied load and crack lenght with the triaxiality of the stresses at the crack tip. In the second case where $\sigma_{x x}=100 \mathrm{MPa}$ and varied $\sigma_{y y}$ (Fig. 7b), a hyperbolic variation has been noticed. The absolute value of the biaxiality parameter $\beta$ decreases with the increase of the applied load and crack length.

In the case of varied bi-equiaxial tensile load (Fig. 7c), it can be seen that the biaxiality parameter increases with the increase of the crack length, and almost constant evolution as a function of the applied load has been observed. In general, the maximum value of the biaxiality parameter $\beta$ does not exceed 0.4 for $a=25 \mathrm{~mm}$. This can be attributed to the neglected effect of the biaxility in the case of a bi-equiaxial loading of the cracked CS.

\section{Influence of crack orientation in the mixed mode}

To investigate the effect of crack orientation on fracture parameters $\left(K_{I}, K_{I I}\right.$, T-stress and biaxiality $\beta$ ) in the mixed mode loading, similar analyses as in the previous Section were carried out for different crack orientations (Fig. 8). All the configurations studied contained an internal central crack of length $2 a$ inclined with respect to the central and horizontal planes of the CS.

(a)

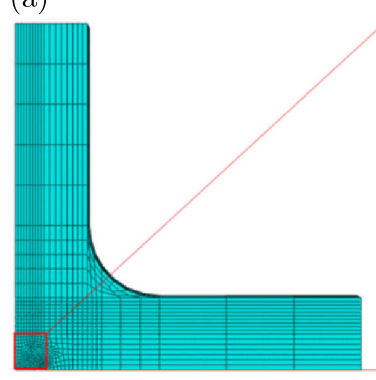

(c)
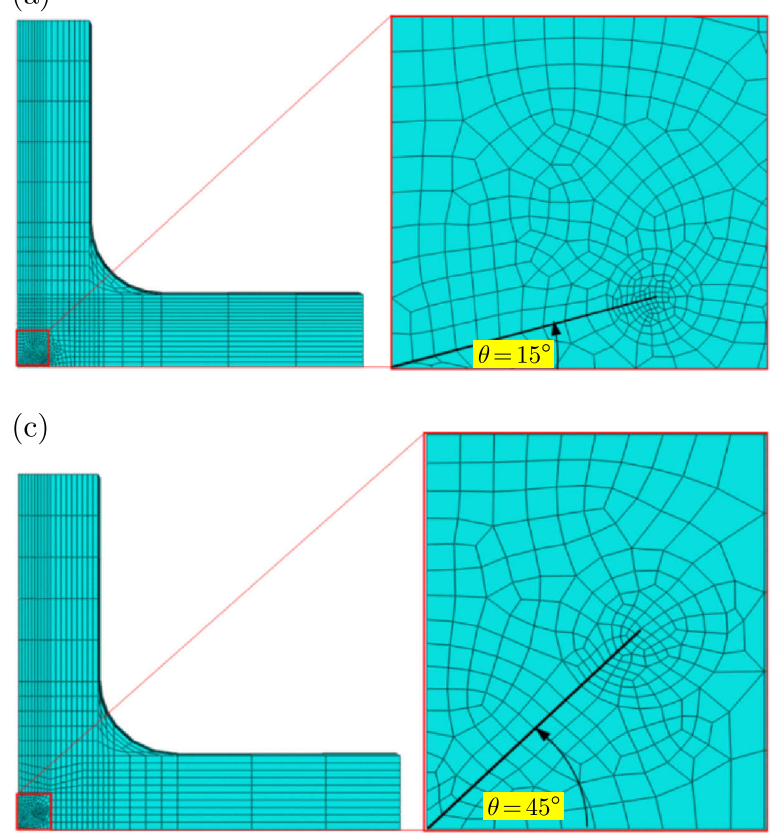

(b)

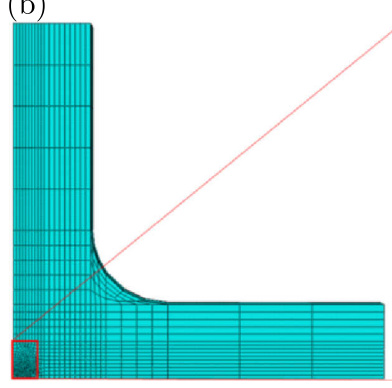

(d)

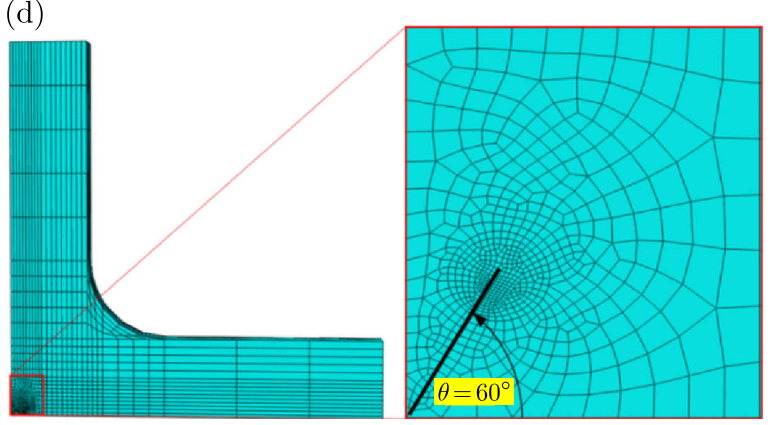

Fig. 8. Illustration of finite element models and details of the refined meshes near the crack tip employed for different crack orientations: (a) $\theta=15^{\circ}$, (b) $\theta=30^{\circ}$, (c) $\theta=45^{\circ}$ and (d) $\theta=60^{\circ}$

In the present investigation, the magnitude of the applied load biaxiality is described by the remote nominal stress ratio $k$. By changing the angle of crack orientation, different combinations of modes I and II can be achieved. The obtained results for each parameter are discussed below.

\subsection{Evolution of SIFs}

In order to make comparison between the obtained results, evolutions of the normalized values of SIF as a function of the applied load in the case of $a=10 \mathrm{~mm}$ with different angles 
of crack orientation ranging from $0^{\circ}$ to $60^{\circ}$ by a step $\Delta \theta=15^{\circ}$ have been plotted in Fig. 9 for different cases of loading.

(a)

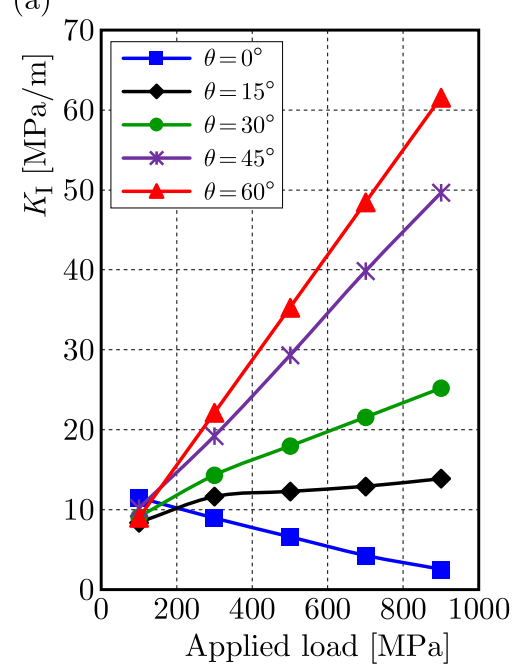

(b)

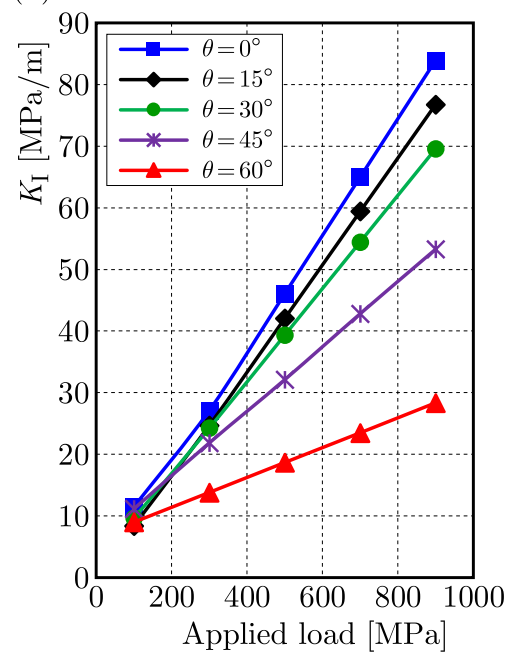

(c)

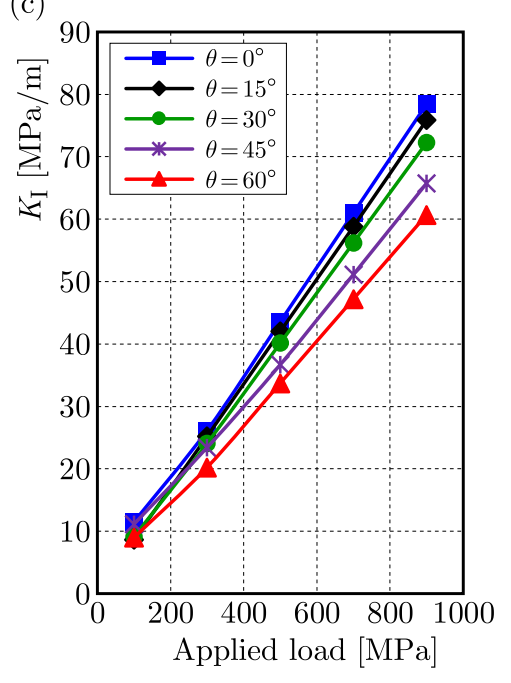

Fig. 9. Evolution of $K_{I}$ as a function of the applied load for different crack angles in the case of:

(a) $\sigma_{y y}=100 \mathrm{MPa}$ and varied $\sigma_{x x}$, (b) $\sigma_{x x}=100 \mathrm{MPa}$ and varied $\sigma_{y y}$, (c) with varied bi-equiaxial tensile load

In the case of $\sigma_{y y}=100 \mathrm{MPa}$ and varied $\sigma_{x x}$ (Fig. 9a), three different types of behavior have been highlighted. When $\theta=0^{\circ}$, the SIF decreases linearly with the increase of the horizontal applied load $\sigma_{x x}$. When $\theta=15^{\circ}$, almost constant evolution has been displayed. However, when $\theta$ is greater or equal to $30^{\circ}$, the latter increases with the increase of $\sigma_{x x}$. In the first behavior, the decrease of $K_{I}$ can be justified by the fact that the displacements of the crack lips are parallel to the applied load $\sigma_{x x}$ (the closure mode is favored). The increase of this parameter in the third behavior is attributed to the opening mode of the crack due to orientation of the applied load $\sigma_{x x}$ with respect to the crack plane which tends towards perpendicularity.

In the case of $\sigma_{x x}=100 \mathrm{MPa}$ and varied $\sigma_{y y}$ (Fig. 9b), it can be seen that the SIF increases linearly with the increase of the vertical applied load $\sigma_{y y}$. This can be attributed to the applied load which is always perpendicular to the crack lips. Indeed, the inclination slope of the variation of $K_{I}$ is much more pronounced for $\theta$ less or equal to $15^{\circ}$ compared to that of $\theta \geqslant 30^{\circ}$.

In the case of varied bi-equiaxial tensile load (Fig. 9c), it can be noted that the SIF increases linearly with the increase of the applied load. In addition, when $\theta$ increases, $K_{I}$ decreases.

Figure 10 shows the evolution of the SIF $K_{I I}$ as a function of the applied load for three different loading conditions and different angles of crack orientation. A crack length of $20 \mathrm{~mm}$ has been set for all the simulation tests.

In the first case (Fig. 10a), it can be observed that the SIF $K_{I I}$ remains constant and equals zero with the increase of the vertical applied load when $\theta=0^{\circ}$. However, when $\theta$ is greater or equal to $15^{\circ}$, the SIF $K_{I I}$ increases linearly with the increase of the vertical applied load. In addition, it can be seen that when $\theta=60^{\circ}$, the obtained values of $K_{I I}$ are lower than those obtained for $\theta=45^{\circ}$. This is due to the fact that in this position $\left(\theta>45^{\circ}\right)$, the crack is solicited to the opening rather than the sliding mode.

In the second case (Fig. 10b), $K_{I I}$ increases with the increase of the applied load. Indeed, when $\theta=0^{\circ}$, an almost constant evolution was noted ( $K_{I I}$ close to 0 ), however, for other values of $\theta, K_{I I}$ varies linearly with the increase of the load except for $\theta=60^{\circ}$, where the slope of $K_{I I}$ variation is lower than that for of $30^{\circ}$ and $45^{\circ}$. This may be justified also by the fact that the shear movement of the crack lips decreases when the orientation angle of the crack exceeds $45^{\circ}$. 
In the third case of the varied bi-equiaxial tensile load (Fig. 10c), three cases were found: (i) when $0^{\circ} \leqslant \theta \leqslant 30^{\circ}, K_{I I}$ increases with the increase of the applied load and decreases with the increase of the crack orientation angle; (ii) when $\theta=45^{\circ}, K_{I I}$ remains equal to 0 with the increase of the applied load; (iii) when $\theta=60^{\circ}$, a slight increase of $K_{I I}$ has been noted but with values lower than those obtained for $0^{\circ} \leqslant \theta \leqslant 30^{\circ}$.

(a)

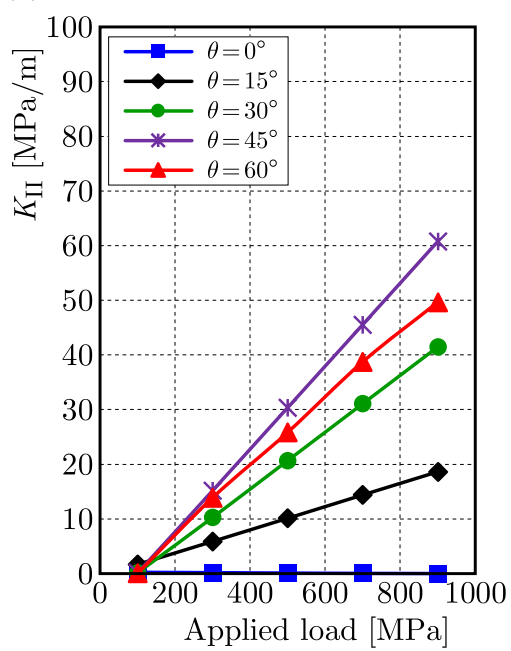

(b)

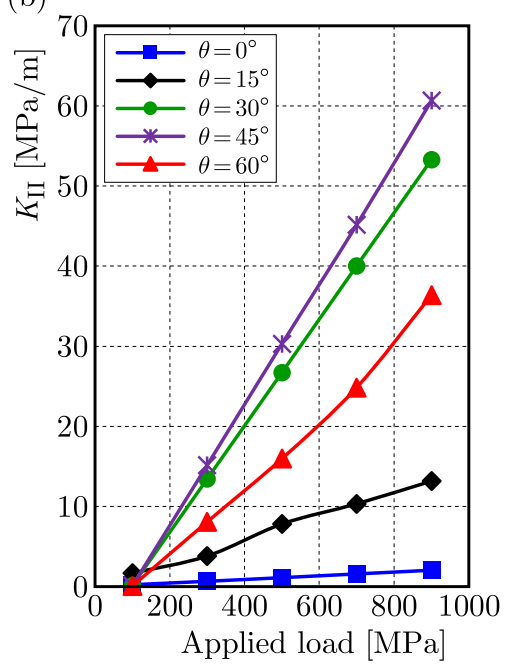

(c)

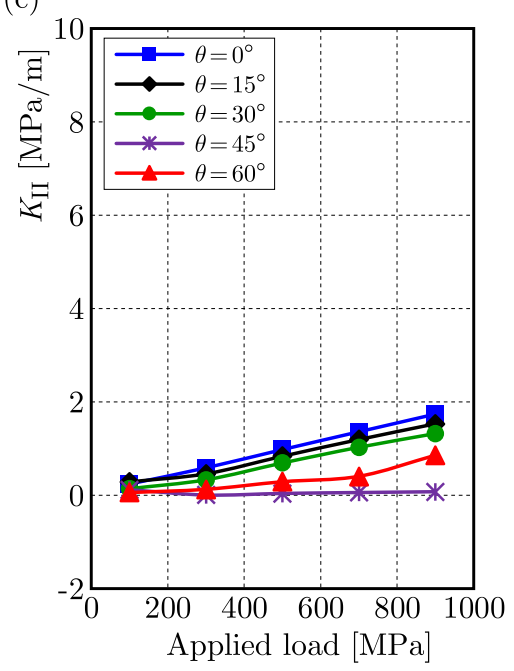

Fig. 10. Evolution of $K_{I I}$ versus the applied load for different crack angles in the case of:

(a) $\sigma_{y y}=100 \mathrm{MPa}$ and varied $\sigma_{x x}$, (b) $\sigma_{x x}=100 \mathrm{MPa}$ and varied $\sigma_{y y}$, (c) with varied bi-equiaxial tensile load

\subsection{Evolution of T-stress}

Figure 11 shows the evolution of the T-stress as a function of the applied loads for different angles of crack orientation $\left(\theta=0^{\circ}, 15^{\circ}, 30^{\circ}, 45^{\circ}\right.$ and $\left.60^{\circ}\right)$ when the crack length is $a=10 \mathrm{~mm}$. For each case, we can draw the following conclusions.

In the case of $\sigma_{y y}=100 \mathrm{MPa}$ and varied $\sigma_{x x}$ (Fig. 11a), we can distinguish three types of variations as a function of $\theta$. When $0^{\circ} \leqslant \theta \leqslant 30^{\circ}$, the T-stress increases linearly with the increase of the horizontal applied load $\sigma_{x x}$ and decreases with the increase of $\theta$. When $\theta=45^{\circ}$, the T-stress remains constant and equal to zero with the increase of the applied load. When $\theta=60^{\circ}$, the T-stress increases linearly in absolute values with the increase of the horizontal applied load.

In the case of $\sigma_{x x}=100 \mathrm{MPa}$ and varied $\sigma_{y y}$ (Fig. 11b), the absolute values of the T-stress increases linearly with the increase of the vertical applied load $\sigma_{y y}$ and decreases with the increase of $\theta$. This can be attributed to the combination effects of the applied load and the crack orientation angle.

In the case of the varied bi-equiaxial tensile load (Fig. 11c), two different types of behavior have been observed: for $\theta=0^{\circ}$ and $15^{\circ}$, the T-stress is positive and increases with the increase of the applied load and the angle $\theta$. On the other hand, for $\theta=30^{\circ}, 45^{\circ}$ and $60^{\circ}$, the T-stress is negative and increases in the absolute value with the increase of the applied load and the angle $\theta$. This change in direction may also be due to the combined effects of the evolution of the applied load with the orientation of the crack.

\subsection{Evolution of the biaxiality parameter}

The biaxiality parameter $\beta$ can provide a good base for estimating the level of scatter in apparent toughness values as a function of the biaxial load ratio $k$ and crack orientation angle $\theta$. 
(a)

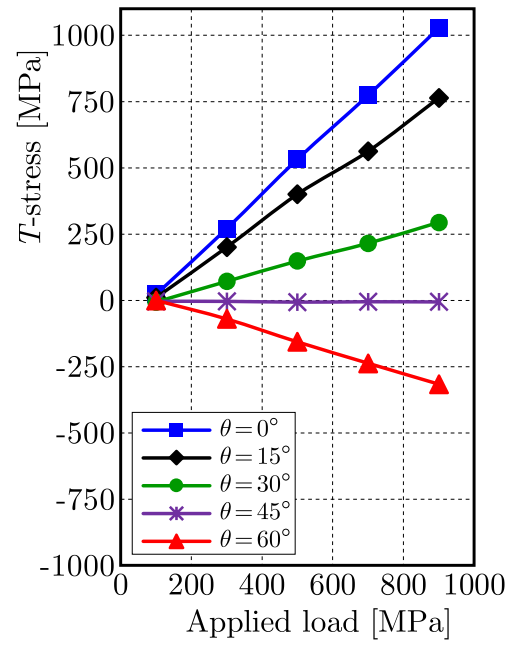

(b)

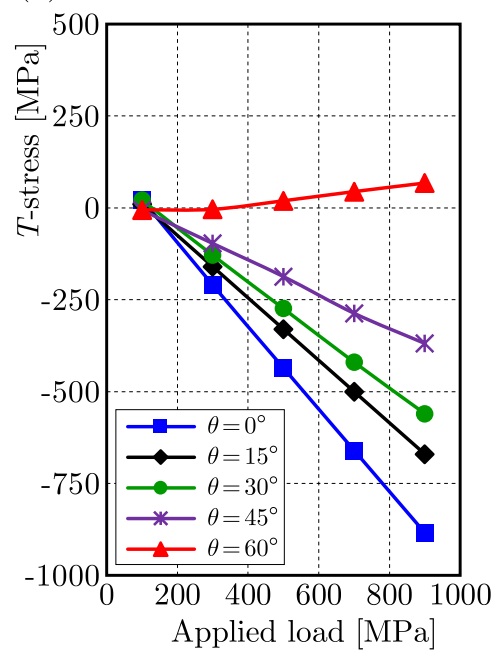

(c)

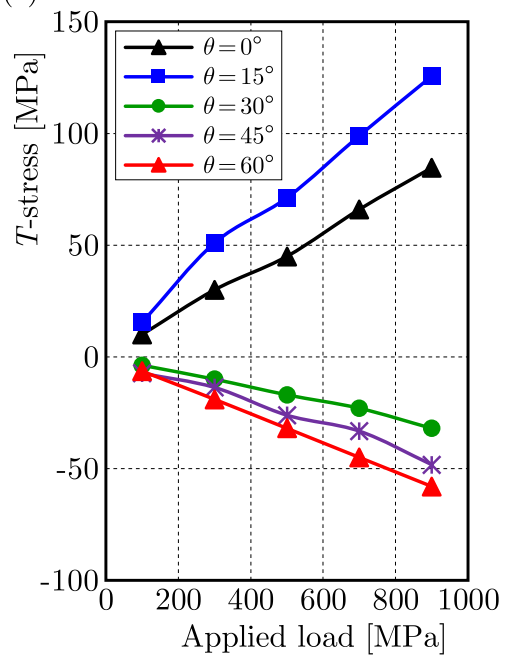

Fig. 11. Evolution of the T-stress versus the applied load for different crack angles in the case of: (a) $\sigma_{y y}=100 \mathrm{MPa}$ and varied $\sigma_{x x}$, (b) $\sigma_{x x}=100 \mathrm{MPa}$ and varied $\sigma_{y y}$ and (c) with varied bi-equiaxial tensile load

In this context, this part of the present work is devoted to study the evolution of the biaxiality parameter $\beta$ under the same tensile loading conditions as previously, and with the same crack length $(a=10 \mathrm{~mm})$. The obtained results are depicted quantitatively in Fig. 12.

(a)

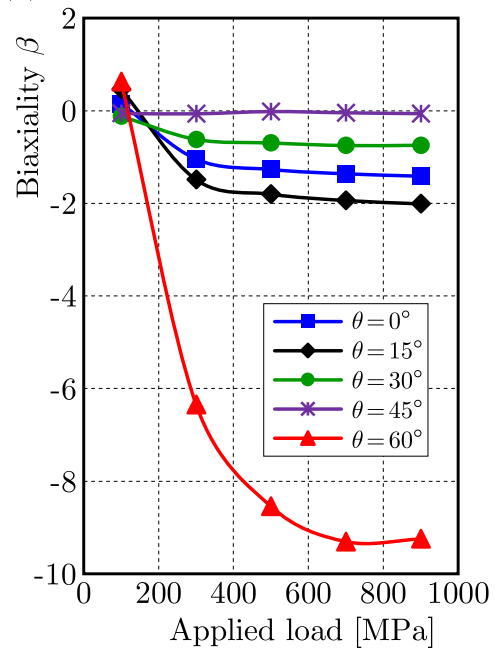

(b)

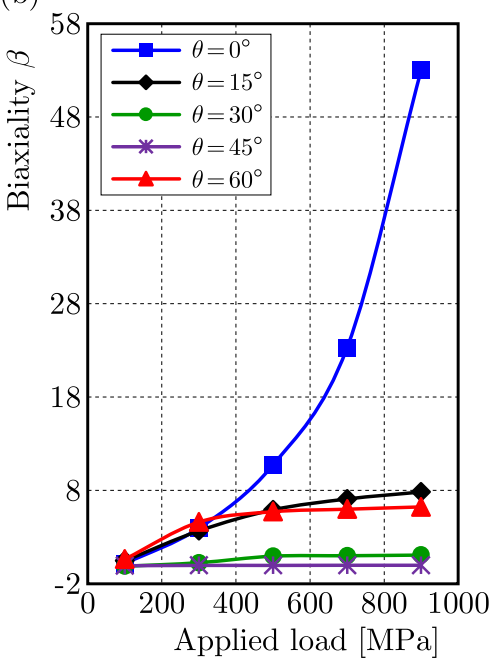

(c)

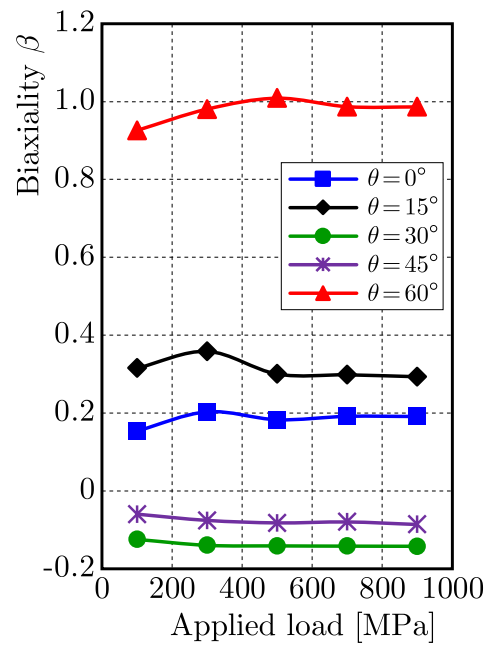

Fig. 12. Evolution of the biaxiality paramter $\beta$ versus loading for different crack orientation angles in the mixed mode in the case of: (a) $\sigma_{y y}=100 \mathrm{MPa}$ and varied $\sigma_{x x}$, (b) $\sigma_{x x}=100 \mathrm{MPa}$ and varied $\sigma_{y y}$, (c) with varied bi-equiaxial tensile load

In the first case of the load ratio $k>1$ with $\sigma_{y y}=100 \mathrm{MPa}$ and $\sigma_{x x}$ varied (Fig. 12a). Two behavior types were observed. The first was noticed for $\theta=0^{\circ}$ where the biaxiality parameter increases with the increase of the load in a parabolic manner. The second is remarked for other values of $\theta$, where $\beta$ increases with the increase of the load up to $300 \mathrm{MPa}$, then it remains constant until the end of loading. In the case of the load ratio $k<1$ with $\sigma_{x x}=100 \mathrm{MPa}$ and $\sigma_{y y}$ varied (Fig. 12b), different behavior has been found.

For $\theta$ between $0^{\circ}$ and $30^{\circ}$, a small increase in the absolute value was observed for a load less than or equal to $300 \mathrm{MPa}$, then quasi-constant behavior was noted when the applied load exceeded $300 \mathrm{MPa}$. For $\theta=45^{\circ}$, the biaxiality parameter was found constant and equal to 
zero. For $\theta=60^{\circ}, \beta$ increased in the absolute value with the increase of the applied load in a hyperbolic way.

In the case of the load ratio $k=1$ with a varied bi-equiaxial tensile load (Fig. 12c), a mostly constant evolution was noticed. In general, values of the biaxiality parameter $\beta$ are between -0.1 (for $\theta=30^{\circ}$ ) and 0.65 (for $\theta=60^{\circ}$ ). This implies the neglected effect of the load ratio $(k=1)$ on the evolution of $\beta$ parameter in the case of cracked CS.

\section{Conclusion}

In this paper, a numerical evaluation of the fracture parameters such as $K_{I}$ and $K_{I I}$, the T-stress and the biaxiality parameter $\beta$ using the finite element method was developed for cruciform specimens under different loading conditions. Based on the obtained numerical results, the following conclusions can be drawn.

- In the case of different horizontal crack lengths:

- The SIFs $K_{I}$ and $K_{I I}$ decrease linearly with the increase of the horizontal applied load $\sigma_{x x}$ and increase linearly with the increase of the vertical applied load $\sigma_{y y}$ or the load ratio $k$.

- The absolute values of the T-stress increase linearly with the increase of the applied load $\sigma_{x x}$ or $\sigma_{y y}$. However, a low increase has been noticed with the increase of the load ratio.

- When $\sigma_{y y}$ is fixed at $100 \mathrm{MPa}$ and $\sigma_{x x}$ is varied from 100 to $900 \mathrm{MPa}$, the biaxiality parameter $\beta$ increases in a parabolic way, however, when $\sigma_{x x}$ is fixed at $100 \mathrm{MPa}$ and $\sigma_{y y}$ is varied, this parameter decreases in a hyperbolic manner. On the other hand, almost a constant evolution has been observed in the case of the varied bi-equiaxial tensile load.

— In the case of different crack angles:

- The SIFs $K_{I}$ and $K_{I I}$ increase linearly with the increase of the biaxial applied load except when $\theta$ is equal to $0^{\circ}$ and $45^{\circ}$. When $\theta=0^{\circ}$, for the first case of loading, $K_{I}$ decreases linearly with the increase of the horizontal applied load $\sigma_{x x}$. When $\theta=45^{\circ}$, for the third case of loading, $K_{I I}$ remains constant and equal to zero with the increase of the bi-equiaxial tensile load.

- When $\sigma_{y y}$ is fixed at $100 \mathrm{MPa}$ and $\sigma_{x x}$ is varied from 100 to $900 \mathrm{MPa}$, three types of behavior have been distinguished for the T-stress as a function of $\theta$ : (i) an increase with positive values for $0^{\circ} \leqslant \theta \leqslant 30^{\circ}$, (ii) the T-stress equal to zero for $\theta=45^{\circ}$ and (iii) a decrease with negative values for $\theta=60^{\circ}$. When $\sigma_{x x}$ is fixed at $100 \mathrm{MPa}$ and $\sigma_{y y}$ is varied, the absolute values of this parameter increases linearly with the increase of the vertical applied load $\sigma_{y y}$. On the other hand, in the case of the varied equi-biaxial tensile load, two different types of behavior have been observed: for $\theta=0^{\circ}$ and $15^{\circ}$, the T-stress is positive and increases with the increase of the applied load, and for $30^{\circ} \leqslant \theta \leqslant 60^{\circ}$, the T-stress is negative and decreases with the increase of the applied load.

- In overall, the biaxiality parameter $\beta$ remains almost constant with the increase of the biaxial applied load except when $\theta=0^{\circ}$. Indeed, $\beta$ increases in a parabolic manner with the increase of the horizontal applied load $\sigma_{x x}$, and when $\theta=60^{\circ}, \beta$ decreases in a hyperbolic way with the increase of the vertical applied load $\sigma_{y y}$.

\section{Acknowledgments}

The authors gratefully acknowledge the scientific support of the two research teams of the Mechanical Laboratory of Lille (LML) (France) and of the Laboratory of Applied Biomechanics and Biomaterials (LABAB) of Oran (Algeria). 


\section{References}

1. ABD-Elhady A.A., 2014, 3D Finite element analysis of plate with small notch subjected to biaxial loading, Emirates Journal for Engineering Research, 19, 1, 27-35

2. Ayatollahi M.R., Dehghany M., Nejati M., 2011, Fracture analysis of V-notched components - Effects of first non-singular stress term, International Journal of Solids and Structures, 48, $1579-1589$

3. Ayatollahi M.R., Pavier M.J., Smith D.J., 1998, Determination of T-stress from finite element analysis for mode I and mixed mode I/II loading, International Journal of Fracture, 91, 283-298

4. Betegon C., Hancock J.W., 1991, Two-parameter characterization of elastic-plastic crack-tip fields, Journal of Applied Mechanics, 58, 104-110

5. Banerjee D., Iadicola M., Creuziger A., Foecke T., 2015, An experimental and numerical study of deformation behavior of steels in biaxial tensile tests, The Minerals, Metals and Materials Society. TMS2015 Supplemental Proceedings, John Wiley \& Sons, Inc., Hoboken, USA

6. Citarella R., Lepore M.A., MalignA., Shlyannikov V.N., 2015, FEM simulation of a crack propagation in a round bar under combined tension and torsion fatigue loading, Frattura and Integrità Strutturale, 31, 138-147

7. Dawicke D.S., Pollock W.D., 1997, Biaxial testing of 2219-T87 aluminum alloy using cruciform specimens, NASA Contractor Report 4782 Analytical Services and Materials, Inc. Hampton, Virginia National Aeronautics, 1-38

8. Eftis J., Subramonian N., 1978, The inclined crack under biaxial load, Engineering Fracture Mechanics, 10, 43-67

9. Freitas M., Reis L., Claudio R., 2014, Mutltiaxial fatigue under biaxial cruciform specimens, Annal Mechanics Fracture, 31, 441-446

10. Gdoutos E.E., 2005, Fracture Mechanics: An Introduction, Second Edition, Springer Netherlands

11. Hatanaka K., Motozawa N., Ogawa H., Sasaki N., Rokukawa S., 1997, A study on fatigue crack growth under biaxial loading using cruciform specimen, 5th International Conference on Biaxial/Multiaxial Fatigue and Fracture, Cracow'97, Poland, Technical University of Opole, 59-76

12. Khelil F., Belhouari M., Aour B., Benseddiq N., 2017, On the efficiency of the numerical evaluation of fracture parameters using a virtual strain gage method, Journal of the Brazilian Society of Mechanical Sciences and Engineering, 39, 589-599

13. Lamkanfi E., Paepegem W.V., Degrieck J., Ramault C., Makris A., van Hemelrijck D., 2010, Strain distribution in cruciform specimens subjected to biaxial loading conditions. Part 2: Influence of geometrical discontinuities, Polymer Testing, 29, 132-138

14. LEEVERS P.S., RAdON J.C., 1982, Inherent stress biaxiality in various fracture specimen geometries, International Journal of Fracture, 19, 311-325

15. Muhsin J.J., Skaker S.H., Kahtan Y.Y., 2013, Experimental investigations of the cruciform specimen of GFRP16 under biaxial monotonic and cyclic loading, International Journal of Science Engineering Research, 4, 12, 494-501

16. Nakamura T., Parks D.M., 1992, Determination of elastic T-stress along three-dimensional crack fronts using an interaction integral, International Journal of Solids and Structures, 29, 13, $1597-1611$

17. Navarro-Zafra J., Curiel-Sosa J.L., Serna Moreno M.C., 2016, Three-dimensional static and dynamic analysis of a composite cruciform structure subjected to biaxial loading: a discontinuum approach, Applied Composite Materials, 23, 2, 139-154

18. Nevalainen M.J., 1997, The effect of specimen and flaw dimensions on fracture toughness, PhD Dissertation, Helsinki University of Technology, Finland 
19. Novotný L., 2012, Calculation of T-stress on 3D specimens with crack, Procedia Engineering, 48, 489-494

20. Papadopoulos G.A., 1988, Crack initiation under biaxial loading, Engineering Fracture Mechanics, 29, 5, 585-598

21. PickARD A.C., 1986, The Application of 3-Dimensional Finite Element Methods to Fracture Mechanics and Fatigue Life Prediction, EMAS, Warley

22. PiCKARD A., 2015, Fatigue crack propagation in biaxial stress fields, Journal of Strain Analysis for Engineering Design, 50, 1, 25-39

23. Rice J.R., 1974, Limitations to the small scale yielding approximation for crack tip plasticity, Journal of the Mechanics and Physics of Solids, 22, 17-26

24. Shlyannikov V.N., 2013, T-stress for crack paths in test specimens subject to mixed mode loading, Egineering Fracture Mechanics, 108, 3-18

25. Shlyannikov V.N., Tumanov A.V., Zakharov A.P., 2014, The mixed mode crack growth rate in cruciform specimens subject to biaxial loading, Theoretical and Applied Fracture Mechanics, 73, 68-81

26. Shlyannikov, V.N., Zakharov, A.P., 2014, Multiaxial crack growth rate under variable T-stress, Engineering Fracture Mechanic, 123, 86-99

27. Sin G.C., 1966, On the Westergaard method of crack analyses, Journal of Fracture Mechanics, 2 , $4,628-631$

28. Truchon M., Amestoy M., DAng-VAn K., 1981, Experimental stydy of fatigue crack growth under biaxial loading, 5th International Conference on Fracture, 4, 1841-1849

29. Upadhyay M.V., Panzner T., van Petegem S., van Swygenhoven H., 2017, Stresses and strains in cruciform samples deformed in tension, Experimental Mechanics, 57, 6, 905-920

30. Williams M.L., 1957, On the stress distribution at the base of a stationary crack, Journal of Applied Mechanics, 24, 109-114

31. www.techniques-ingenieur.fr, Charactéristiques mécaniques, ALMET p57.0

32. Xiao R., Li X., Lang L., Chen Y., Ge Y., 2015, Design of biaxial tensile cruciform specimen based on simulation optimization, 2nd International Conference on Machinery, Materials Engineering, Chemical Engineering and Biotechnology (MMECEB 2015) 